\title{
Why deterministic traffic shows the highest reordering ratio
}

\author{
Sebastian Gunreben \\ Universität Stuttgart, IKR \\ Pfaffenwaldring 47 \\ Stuttgart, Germany \\ Email: gunreben@ikr.uni-stuttgart.de
}

\author{
Óscar González de Dios \\ Telefónica I+D \\ Emilio Vargas 6 \\ Madrid, Spain \\ Email: ogondio@tid.es
}

\begin{abstract}
In optical burst switching networks, bursts arrive out-of-sequence due to contention resolution schemes as well as contention avoidance schemes. Out-of-sequence bursts may imply out-of-sequence packets, which affect the packet layer performance. Consequently, it is necessary to classify and investigate the resulting burst/packet out-of-sequence pattern. In our previous work, we presented an analytic model to evaluate the out-ofsequence pattern for deterministic traffic showing constant interarrival times using the IETF WG IPPM reordering metrics. This paper extents our previous work. If the amount of out-of-order arrivals is small, the performance impact may also be small. One indication of the amount of out-of-order arrivals is the reordering ratio. In this paper, we prove analytically that deterministic traffic with constant inter-arrival time generates the highest reordering ratio for our model with respect to any other traffic characteristic. Consequently, deterministic traffic serves as an upper bound for the estimation of the reordering ratio. It allows a quick estimation of the reordering ratio and indicates if further investigations on the reordering pattern are required.
\end{abstract}

Index Terms-burst reordering, worst case considerations, deterministic traffic

\section{INTRODUCTION}

Optical burst switching (OBS, [1]) is a promising network technology for core and metro networks based on wavelength division multiplex. At the OBS network edge, the OBS assembly unit aggregates packets based on their destination address and optionally their service class. At the end of the assembly process, the assembly unit forwards the burst to the optical transmission unit heading to the destination node.

Literature proposes various assembly schemes like time- or size-based assembly or a combination of both. Each of these schemes shows a different traffic characteristic of the departing bursts depending on the input traffic.

In OBS networks, contention occurs on intermediate nodes if two or more bursts request the same wavelength at the same time. Given this situation, original OBS discards all but one successful burst. These burst losses degrade the transport service and stimulate the research on contention resolution and contention avoidance schemes to reduce burst losses [2]-[5].

Both, contention resolution schemes and contention avoidance schemes delay bursts compared to the primarily planned shortest path. As a result, the burst order at the destination may change resulting in out-of-sequence arrivals. Since each data burst is an aggregate of multiple packets, out-of-sequence burst arrivals also imply a special out-of-sequence packet arrival, which may affect transport and application layer protocols.

Transport protocols provide an unreliable or a reliable connection service to applications. Out-of-sequence packets of the same flow affect the performance of these protocols. [6] gives an overview on this topic. As summarized in [7], literature discusses these impacts on the transport layer in several publications. The transmission control protocol (TCP, [8]) is the most important representative of transport protocols for a reliable connection service in IP-based networks. The basic TCP congestion control algorithm [8] suffers from missing or out-of-sequence packets. Literature extensively studies in [9][14] the impact of burst losses on TCP.

Literature rarely studies the impact of burst reordering on TCP and other upper-layer protocols. In our previous work [7], we presented an analytic model as well as a new methodology to study burst reordering pattern in OBS networks. Perelló et al. in [15] as well as Schlosser in [16] focus on burst reordering using simulations rather than formal methods. Their simulation models usually include several layers in the simulation not allowing a deep in-sight in the reordering process.

A more basic problem is the definition of an out-of-sequence burst and the characterization of its out-of-sequence pattern. Literature proposes several different out-of-sequence metrics. Piratla et al. propose in [17] the reorder density to measure the amount of reordered packets and the displacement of a single packet. They compare their approach to the standardized metrics of the IETF in [18]. Also in the field of optical burst reordering, Callegati et al. propose a simple measure for outof-sequence bursts in [12]. Both metrics lack a standardized approach. For a comprehensible study, we consider the standardized metrics of the IETF WG IPPM [19].

In our previous work [7], [20], [21], we proposed a first model to investigate the burst reordering phenomena analytically and showed its applicability for traffic types showing deterministic traffic with a constant inter-arrival time. We estimated these calculations as a worst-case scenario regarding the reordering ratio but did not provide a formal proof. This paper closes this gap and provides an analytic proof, showing that the reordering ratio (the amount of out-of-sequence arrivals) reaches its maximum with deterministic traffic.

Starting from a given reordering model, our findings enable 
a quick estimation of the amount of reordered bursts and packets at the destination. Thereby, our findings enable a worst-case estimation of the amount of reordered bursts. The amount of out-of-sequence bursts/packets indicates if an indepth investigation on the reordering pattern is necessary or not [19]. Applying formal methods requires no multi-layer network simulations. In this scenario, single layer studies on the OBS layer are sufficient, a simulation of the whole network stack ranging from the transport protocol down to the OBS network layer is not necessary.

We structure our paper in the following way: In section II we introduce the IETF definition of reordered bursts/packets and the reordering ratio. Section III reviews the reordering ratio for deterministic traffic schemes and section IV proves our findings with respect to generic traffic characteristics. Section V summarizes our work.

\section{REORDERING METRIC}

This section introduces the definition of IP packet reordering, which is consistent with the proposal of the IETF WG IPPM [19]. This definition also holds for generic packetswitched networks like OBS networks.

The reordering definition includes the following theoretic considerations. The source node assigns each burst a sequence number. The sequence numbers increase strict monotonically. At the destination node, a 3-tuple $\left(i, s[i], s^{\prime}[i]\right)$ characterizes each burst arrival. Index $i$ indicates the arrival order at the destination. $s[i]$ denotes its sequence number and $s^{\prime}[i]$ denotes the next expected sequence number at this arrival instance.

The previously received burst determines the value of $s^{\prime}[i]$. We distinguish two cases:

1) $s[i]<s^{\prime}[i]$ burst $i$ arrives out-of-sequence and $s^{\prime}[i+1]:=s^{\prime}[i]$.

2) $s[i] \geq s^{\prime}[i]$ burst $i$ arrives in order and $s^{\prime}[i+1]:=s[i]+1$.

Literally, a burst gets to the destination out-of-sequence, if there is one burst with a larger sequence number arriving prior to it. For instance, burst $I$ shows sequence number $i$ and burst $J$ shows sequence number $j$ where $i<j$. Burst $I$ leaves the source node earlier than burst $J$. The inter-departure time ${ }^{1}$ between burst $I$ and burst $J$ is the random variable $T_{I J}$. The path from source to destination shows for each burst a different delay $D$, where $D \geq 0$. Burst $I$ receives random delay $D_{I}$, while burst $J$ receives a random delay of $D_{J}$. According to the definition, burst $I$ arrives out-of-sequence with respect to burst $J$ if the following inequality holds:

$$
D_{I}>D_{J}+T_{I J}, \quad \text { where } D_{I} \geq 0, D_{J} \geq 0, T_{I J}>0
$$

With this definition, [19] derives the reordering ratio indicating the proportion of bursts, which arrive out-of-sequence. The reordering ratio equals the probability of an out-ofsequence burst arrival at the destination. As shown in [7], the burst reordering ratio equals the packet reordering ratio.

\footnotetext{
${ }^{1}$ time between two burst departures
}

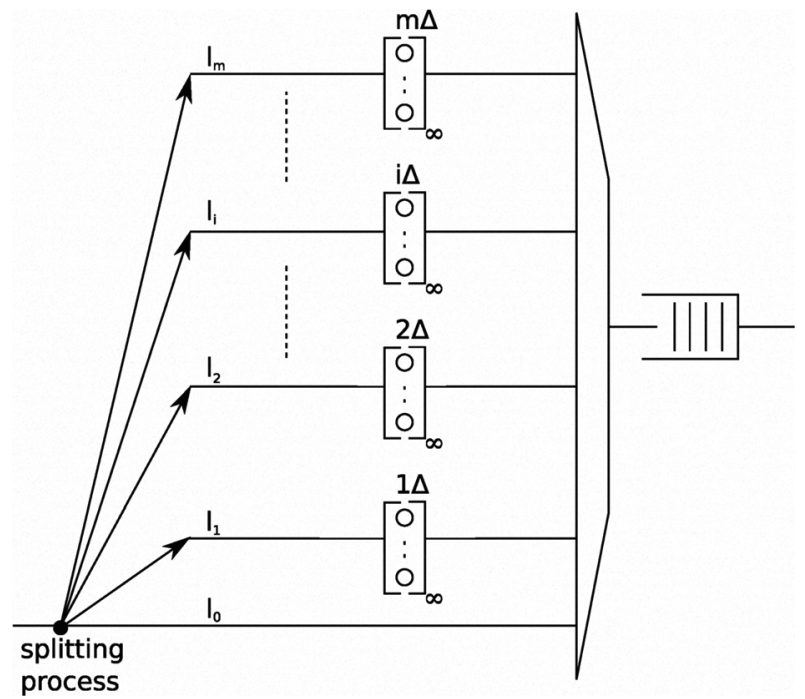

Fig. 1. Queueing model

\section{REORDERING ANALYSIS}

This section first reviews our reordering model and second shows the methodology to obtain the reordering ratio for deterministic traffic showing constant inter-departure times.

\section{A. Reordering model}

Our reordering model considers bursts sent from an OBS source node to an OBS destination node. These bursts may follow different paths due to implemented contention resolution and avoidance schemes. Consequently, in an end-to-end consideration the bursts show a certain jitter at the destination node. The distribution of the jitter reflects the different paths from source to destination and their frequency of occurrence.

We discretize the delay jitter and model each delay alternative from source to destination node by one abstract link $l$. In general we assume $m \in \mathbb{N}^{+}$parallel abstract links $l_{1}$ to $l_{m}$. Besides this, $l_{0}$ represents the primarily planned shortest path with no extra delay. $m$ is finite as the network itself limits the number of alternative paths.

A burst follows an abstract link and receives an additional delay, reflecting for instance the time in a fibre delay line or on a deflection path. Abstract link $l_{i}$ delays the burst following $l_{i}$ by $\Delta_{i} \in \mathbb{R}^{+}$.

The jitter distribution at the destination node reflects the different probabilities of the abstract links. Consequently, we define the probability $p_{i}$ for a burst following abstract link $l_{i}$. Thereby, the law of total probability holds: $\sum_{k=0}^{m} p_{k}=1$.

Summarizing, a 3-tuple $\left(k, p_{k}, \Delta_{k}\right)$ characterizes each abstract link $l_{k}$ : the link number $k, 0 \leq k \leq m$; the probability $p_{k}, 0 \leq p_{k} \leq 1$ to follow $l_{k}$ and the delay $\Delta_{k}$. Note, as an OBS network switches each burst separately, each burst decides independently of all other bursts which abstract link to follow.

\section{B. Network delay distribution}

The previous section introduced the delay $\Delta_{k}$ of an abstract link $k$. This section motivates the correlation between the inter- 


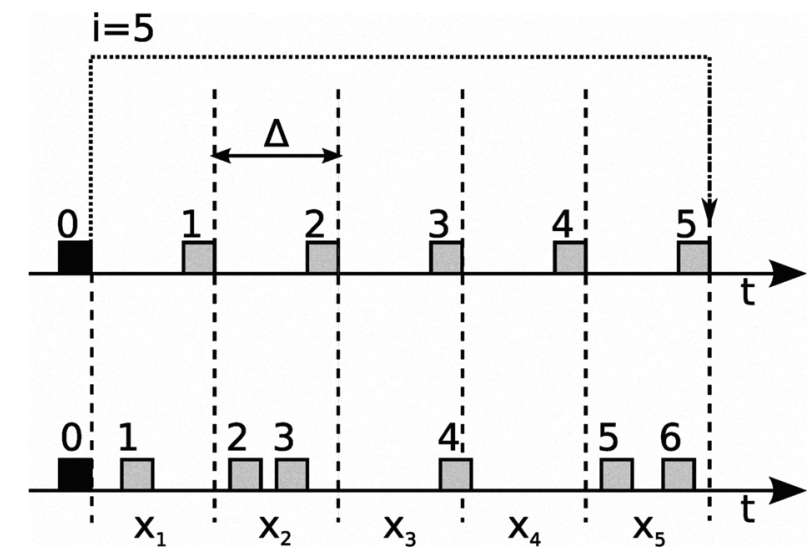

Fig. 2. Reordering with deterministic (top) and generic traffic (bottom) traffic

arrival time and the delay $\Delta_{k}$ for deterministic traffic showing constant inter-arrival times.

In our scenario, we consider deterministic traffic with a constant inter-arrival time. This scenario corresponds to constant bit rate traffic as introduced in our previous work [7]. There, the delay on two neighbouring abstract links differs by exactly the mean inter-arrival time: $\Delta_{i+1}-\Delta_{i}=\Delta=\mathrm{E}\left[T_{I J}\right]$. Consequently, the delay per abstract link $k$ results in $\Delta_{k}=k \Delta$.

Figure 1 depicts the corresponding queuing model of the whole reordering process. This model represents our simulation model used to validate our findings. The bursts leave the source node and enter the reordering model. An initial splitting process assigns each burst to a certain abstract link following the probability distribution of the abstract links. Each abstract link - except $l_{0}$ - delays the burst by $k \Delta$ using an infinite number of server places. After leaving the abstract link the burst leaves the network and arrives at the destination node. The initial splitting process determines the total number of delay units to pass.

\section{The reordering probability}

Our previous work derived the reordering extent and the TCP relevant $n_{r}$-reordering metric in [20] and [7]. Here, we briefly summarize the calculation of the reordering ratio for a deterministic traffic scenario $\mathfrak{C}$, where $\operatorname{VAR}\left[T_{I J}\right]=0$. For this investigation, we assume a lossless burst network.

Figure 2 depicts in the first row the general reordering scenario for one selected burst, i.e., the test burst, but our considerations also hold for any other burst, too. As we consider the reordering probability, we distinguish two kinds of bursts:

1) the test burst (black) for which we evaluate the reordering ratio. Without loss of generality, its sequence number $s$ is $s=0$.

2) bursts (gray) departing later but arriving earlier than the test burst because of the delay of the test burst. These bursts cause the test burst arriving out-of-sequence.

The test burst arrives out-of-sequence at the destination if there is at least one burst arrival with $s>0$ prior to the test burst (at least one out of five bursts of figure 2 arrives before the test burst). The reordering probability is a joint probability of (a) the test burst receives a delay and (b) there is at least one burst arrival with a larger sequence number than zero before the test burst. For condition (a), we assume that the test burst receives a delay of $d_{t}$ with probability $p_{d_{t}}$. Then there are $d_{t}$ candidate bursts, which may accomplish condition (b).

We derive the probability of (b) by its complement, i. e., that there is no arrival before the test burst. The random variable of the delay of the test burst is $D_{t}$. Then the probability that the candidate burst $j, 0<j \leq d_{t}$ does not accomplish condition (b) is $\mathrm{P}\left(B=0\left|D_{t}=d_{t}\right| J=j\right)=\sum_{k=d_{t}-j+1}^{m} p_{k}$. $B$ denotes the random variable of the arrival of burst $j$ before the test burst. The sum of probabilities represents the probability that burst $j$ arrives later than the test burst. It considers the position of the burst $j$ and sums up the probabilities of all abstract link leading to a later arrival than the test burst. For instance, consider figure 2. Burst 3 arrives later than the test burst if it follows abstract links $i \geq 2$. It arrives before the test burst if following abstract links 0 and 1 .

The joint probability that none of the candidate bursts accomplish condition (b) at the same time is $\mathrm{P}\left(B=0 \mid D_{t}=\right.$ $\left.d_{t}\right)=\prod_{j=1}^{d_{t}} \mathrm{P}\left(B=0\left|D_{t}=d_{t}\right| J=j\right)$. The complementary probability of $\mathrm{P}\left(B=0 \mid D_{t}=d_{t}\right)$ accomplishes condition (b). The burst reordering probability for deterministic traffic results in

$$
\operatorname{Pr}(\mathfrak{C})=\sum_{i=1}^{m} p_{i}\left(1-\prod_{k=1}^{i} \sum_{j=i-k+1}^{m} p_{j}\right)
$$

\section{WORST CASE CONSIDERATIONS}

This section proves that the analytic reordering model together with packet arrivals showing constant inter-departure times serve as a worst-case approximation of the expected reordering ratio. We prove that deterministic traffic with a constant inter-arrival time generates a larger reordering ratio than any other traffic.

For the generic traffic scenario, we assume a mean interarrival time of $\mathrm{E}\left[T_{I J}\right]$. The reordering model corresponds to the analytic reordering model of section III-A with $m$ abstract links. According to the definition, an abstract link $i$ delays a packet by $i \Delta=i \mathrm{E}\left[T_{I J}\right]$ with probability $p_{i}$, where $\sum_{i=0}^{m-1} p_{i}=1$. Thereby, the abstract link probability is arbitrarily distributed. With this scenario, we state the following theorem:

Theorem. The reordering ratio of traffic stream $\mathfrak{G}$ showing an arbitrary inter-arrival time distribution with mean $\mathrm{E}\left[T_{I J}\right]$ is less or equal than the reordering probability of a traffic stream $\mathfrak{C}$ showing constant inter-arrival times (VAR $[\mathfrak{C}]=0$ ) with the same mean inter-arrival time $\mathrm{E}\left[T_{I J}\right]$ on the same reordering model. According to (3), this translate to the following proposition:

$$
\operatorname{Pr}(\mathfrak{G}) \stackrel{!}{\leq} \operatorname{Pr}(\mathfrak{C})
$$

Proof: If the traffic characteristic shows deterministic arrival instances and constant inter-arrival times, the time axis 


$$
\begin{aligned}
P(\mathfrak{G}) & =\sum_{i=1}^{m} p_{i}\left(1-\sum_{n=0}^{\infty} \operatorname{Pr}\left(N_{t}=n\right) \sum_{\vec{x} \in S_{n}^{i}} \operatorname{Pr}\left(\vec{X}=\vec{x} \mid N_{t}=n\right) \prod_{k=1}^{i}\left(\sum_{j=i-k+1}^{m} p_{j}\right)\right. \\
& =\sum_{i=1}^{m} p_{i}\left(1-\sum_{n=0}^{\infty} \sum_{\vec{x} \in S_{n}^{i}} \operatorname{Pr}\left(\vec{X}=\vec{x} \wedge N_{t}=n\right) \prod_{k=1}^{i}(\underbrace{\sum_{j=i-k+1}^{m} p_{j}}_{q_{k}})^{x_{k}}\right)
\end{aligned}
$$

shows slotted characteristics as depicted in figure 2. At each slot border, there occurs exactly one burst (first row). The time between two bursts is constant $\Delta$. If the test burst follows abstract link $i$, then in the mean time at maximum $i$ bursts may pass by, if they do not follow any abstract link, causing a later arrival than the test burst. The figure illustrates the scenario for $i=5$, i. e., the test burst follows abstract link 5. Besides for the constant inter-arrival time, the slots serve a different purpose. The slot number determines the sum of probabilities for a later arrival than the test burst (cf. (3)).

Figure 2 shows the scenario for a general burst arrival pattern in the bottom row. Consequently, the number of burst arrivals within one slot varies. Mapping the burst arrivals onto the slotted time axis leads to a random distribution of arrivals within each slot. In the figure, $x_{i}$ denotes the number of burst arrivals within one slot. For instance, in the first slot one burst arrives, while in the third slot no burst arrives. Thereby, we neglect the burst length and consider only the arrival instance of the burst. The distribution of arrivals within the slots corresponds to the burst traffic characteristic.

For the generic traffic scenario, $p_{x}(t)$ is the probability distribution function of the number of arrivals within an arbitrary time interval $t$. Then, for the first slot the following equations hold:

$$
\begin{aligned}
& \sum_{n=0}^{\infty} n p_{n}(\Delta)=1 \\
& \sum_{n=0}^{\infty} p_{n}(\Delta)=1
\end{aligned}
$$

(5) states the requirement of the mean inter-arrival time being $\Delta=\mathrm{E}\left[T_{I J}\right]$ and (6) gives the total probability of all arrivals.

We consider for the test burst the arbitrary delay of $i$. With this, we introduce the vector $\vec{x}$ representing the number of arrivals within the $i$ slots. Therein, $X_{k}$ holds the random number of arrivals within slot $k$, where $1 \leq k \leq i$. Additionally, $S^{i}$ represents the co-domain of $\vec{X}$ holding all possible vectors $\vec{x}$ of length $i$. The subset $S_{n}^{i}$ shows exactly $n$ arrivals within the $i$ slots. We summarize these properties in the following equations:

$$
\begin{aligned}
& \sum_{n=0}^{\infty} \sum_{\vec{x} \in S_{n}^{i}} \operatorname{Pr}(\vec{X}=\vec{x} \wedge N=n)=1 \\
& \sum_{n=0}^{\infty} \sum_{\vec{x} \in S_{n}^{i}} n \operatorname{Pr}(\vec{X}=\vec{x} \wedge N=n)=i
\end{aligned}
$$

(7) states the total probability of all possible arrivals within the next $i$ slots. (8) represents the average number of packets in the whole interval. According to the definition of section III-B this is $i$ for $i$ slots.

According to the reordering probability of equation (3), we formulate an equivalent equation to determine the reordering probability of a general arrival pattern in (2). Its structure follows equation (3).

The outer sum considers all possible branches $i$ of the test burst $\left(p_{i}\right)$. The bracket shows the complementary distribution of a burst arrival with a larger sequence number. The product combines the number of possible arrivals $N$ and the distribution of these arrivals among the $i$ slots $\left(S_{n}^{i}\right)$. For each arrival within a certain slot, the same condition applies for the sum of probabilities (for a later arrival than the test burst) equivalent to (3). The product of sums guarantees the joint probability for all slots. Further, equation (2) reformulates this conditioned probability to a joint probability in the second line. The innermost sum represents a sum of probabilities. For convenience of reading, $q_{k}$ abbreviates the individual factors.

According to the theorem, (4) must hold for any distribution of $p$. As a first step, we enforce our proposition by demanding that each individual summand of (2) is smaller than the corresponding summand of (3). If this is true, also (4) is true.

Applying this simplifications leads to the following inequality, where the expression of $\mathfrak{G}$ (from (2)) stands left and the expression of $\mathfrak{C}$ (from (3)) stands right (the inequality sign changed because of resolving the complementary probability):

$$
\begin{aligned}
& \sum_{n=0}^{\infty} \sum_{\vec{x} \in S_{n}^{i}} \operatorname{Pr}(\vec{X}=\vec{x} \wedge N=n) \prod_{k=1}^{i} q_{k}^{x_{k}} \geq \prod_{k=1}^{i} q_{k} \\
& \sum_{n=0}^{\infty} \sum_{\vec{x} \in S_{n}^{i}} \operatorname{Pr}(\vec{X}=\vec{x} \wedge N=n) \prod_{k=1}^{i} q_{k}^{x_{k}-1} \geq 1
\end{aligned}
$$

The next approximation focus on the $q_{k}^{x_{k}}$ product of equation (9). The product consists of $n$ factors as $\sum_{k=1}^{i} x_{k}=n$. The subsequent step divides both sides by the individual 


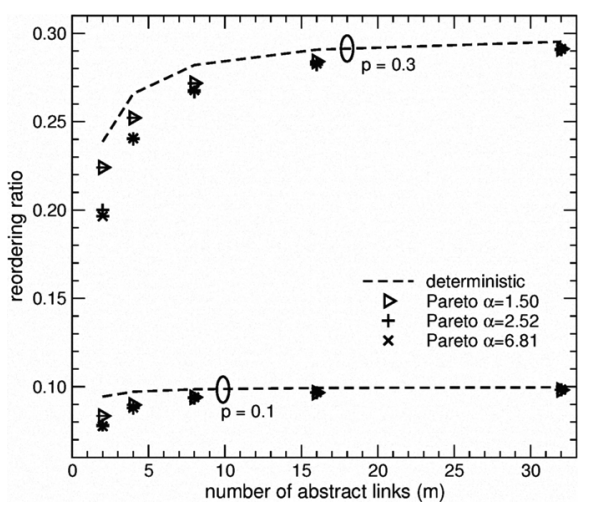

Fig. 3. Geometric distribution, Pareto traffic

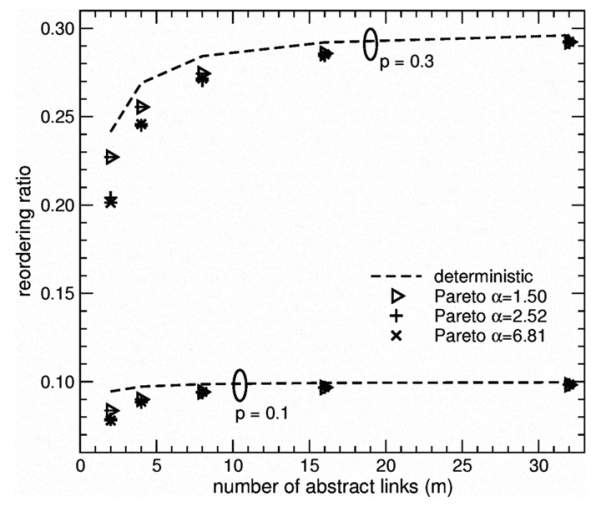

Fig. 4. Uniform distribution, Pareto traffic

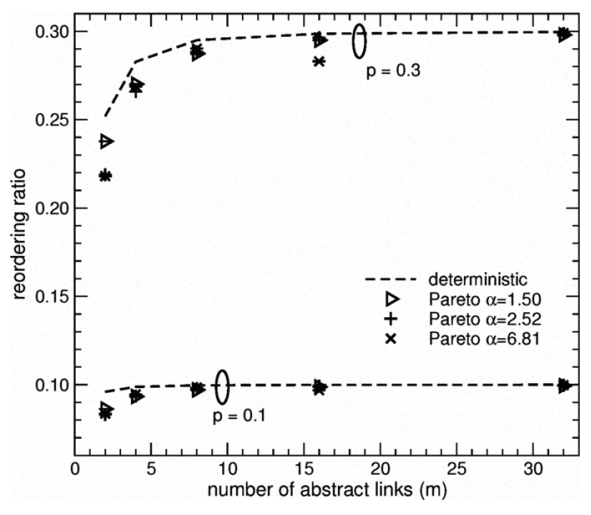

Fig. 5. Linear distribution, Pareto traffic $q_{k} \leq 1$ for $1 \leq k \leq m$. As the product reflects a joint probability, we approximate the individual $q_{k}$ by its smallest value $q=\min _{k}\left\{q_{k}\right\}$. Inserting $q$ in (9) leads to the approximation of the product:

$$
\prod_{k=1}^{i} q_{k}^{x_{k}-1} \geq q^{n-i}
$$

With this approximation, (9) simplifies to:

$$
\sum_{n=0}^{\infty} q^{n} \operatorname{Pr}(\vec{X}=\vec{x} \wedge N=n) \geq q^{i}
$$

With $i=\mathrm{E}[N]$ and $g(n)=q^{n}$ equation (11) becomes:

$$
\underbrace{\sum_{n=0}^{\infty} g(n) \operatorname{Pr}(\vec{X}=\vec{x} \wedge N=n)}_{\mathrm{E}[g(X)]} \geq g(\mathrm{E}[X])
$$

(12) shows the same structure than the inequality by Jensen [22] for convex functions $\varphi$ :

$$
\varphi\left(\frac{\sum a_{i} x_{i}}{\sum a_{i}}\right) \leq \frac{\sum a_{i} \varphi\left(x_{i}\right)}{\sum a_{i}} \quad \text { where } a_{i}>0 .
$$

In our case $\varphi=g(n)=q^{n}$ is convex as $g^{\prime \prime}(n)>0, \forall n>0$.

\section{A. Numerical results}

In this section, we first introduce the applied network delay distributions and traffic characteristics for a comprehensive discussion. Second, we show some illustrative results to visualize the upper bound burst reordering ratio and compare these results to the simulation results of other the traffic characteristics.

1) Parameterization: We parameterize our reordering model with the following parameters: (a) the probability of delay $p$, which corresponds to the complementary probability to follow $l_{0}$, (b) the number of abstract links $m$ and (c) the delay distribution among the $m$ abstract links. We distinguish three different delay distributions, where $i$ gives the index of the abstract link with $1 \leq i \leq m$.

- geometric distribution:

$$
p_{i}=q(1-q)^{i-1} \text { with } q=1-(1-p)^{1 / m} \text {, }
$$

- linear distribution: $p_{i}=2 i p /\left(m^{2}+m\right)$,

- uniform distribution: $p_{i}=1 / m$.

We load our traffic model with three different traffic distributions: Pareto traffic, Poisson traffic and deterministic traffic for comparison. The properties of the Pareto distributed traffic, showing heavy tail characteristics, are:

$$
\begin{aligned}
f_{P}(t) & =\alpha \frac{k^{\alpha}}{k^{\alpha+1}} \\
\mathrm{E}[T] & = \begin{cases}\frac{\alpha k}{\alpha-1} & \text { for } \alpha>1 \\
\infty & \text { for } \alpha \leq 1\end{cases}
\end{aligned}
$$

The first line shows the probability density function, while the second line shows the mean value. The parameters of the Pareto distribution are the shape parameter $\alpha>0$ and the minimum value $k>0$. If $\alpha>2$, then also the variance exists.

The next equations show the characteristics attributes of the well known Poisson distribution (left) and the deterministic distribution (right):

$$
\begin{aligned}
f_{N}(t) & =\lambda \exp (-\lambda t) & f_{D}(t) & =\delta(t-d) \\
\mathrm{E}[T] & =\frac{1}{\lambda} & \mathrm{E}[T] & =d
\end{aligned}
$$

Therein, $\lambda$ represents the mean arrival rate and $d$ represents the constant inter-arrival time.

2) Illustrative results: For numerical results, we fix the traffic mean rate. In case of the negative exponentially distributed inter-arrival time and deterministic traffic, this relates directly to the parameters of the distribution. For the Pareto traffic model, we use three different parameterizations for a wide range of $\alpha$ leading all to the same mean rate: $\alpha_{1}=6.81, k_{1}=$ $0.77 ; \alpha_{2}=2.52, k_{2}=0.59$ and $\alpha_{3}=1.5, k_{3}=\frac{1}{3}$.

Figures 3,4 and 5 show the calculated values of the reordering metric for deterministic traffic (dashed line) and the simulated values for the Pareto distributed traffic for an increasing number of abstract links. We simulated these scenarios with our event-driven simulation library [23]. For reasonable results, we obtained the statistical values from ten batches each including at least one million burst arrivals (the figures also depict the tiny confidence intervals). We obtained the results for the deterministic traffic scenario, by our analytic model from [7]. As these results match the corresponding simulation results, we skip the simulation results here. 


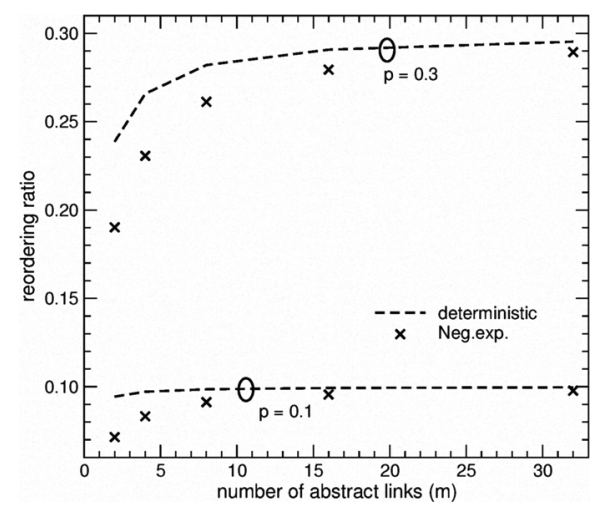

Fig. 6. Geometric distribution, NegExp traffic

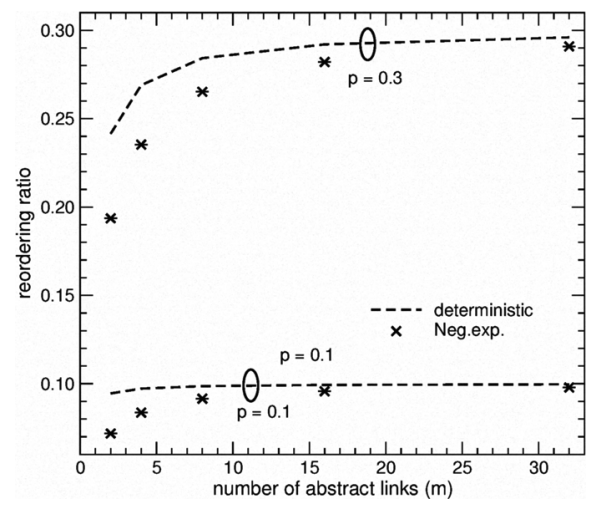

Fig. 7. Uniform distribution, NegExp traffic

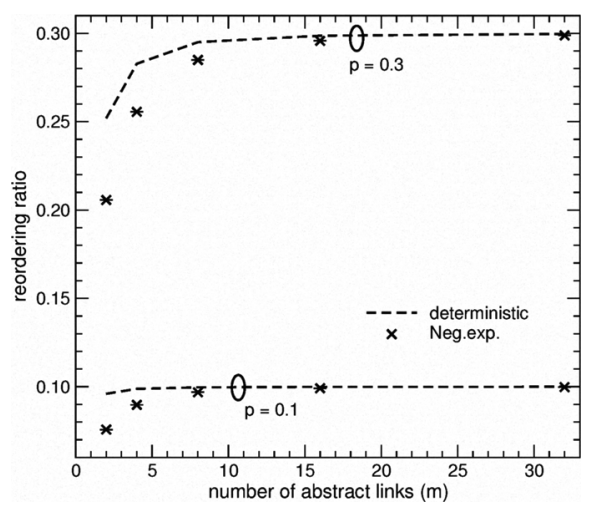

Fig. 8. Linear distribution, NegExp traffic
Our evaluation of the formal proof includes branch probabilities of 0.1 and 0.3 for the introduced three different delay distributions. We also simulated the much lower branch probability of 0.01 but skip the results as they do not provide additional findings.

For a small number of abstract links, the reordering probability in all cases is much lower than the branch probability $p$. With the increasing number of links, the reordering ratio approximates the branch probability of 0.1 and 0.3 , respectively.

The reordering probability of the different values of $\alpha$ is very similar if $\alpha>2$. For $\alpha<2$, the reordering ratio is larger, as the variation is much larger in this case. Nevertheless, the reordering ratio of all Pareto parameterizations is well below the corresponding value of the deterministic traffic.

Thereby, the number of links has a significant impact on the reordering ratio while the network delay distribution has only minor influence. For the geometric, uniform and linear distribution, the values of the reordering ratio are very similar.

Figures 6, 7 and 8 show the same scenario for Poisson traffic showing negative exponentially distributed arrival times. Again, the figures depict the reference value for the deterministic traffic showing the same mean than the Poisson traffic.

The findings for the Poisson traffic are equivalent to the findings of the Pareto traffic. The reordering ratio is always below the deterministic value as well as the reordering value approaches the branch probability for a large number of links.

Comparing the reordering ratio to the corresponding value of the Pareto traffic highlights that Poisson traffic shows in general a smaller reordering ratio than Pareto traffic.

The analytic model with a constant inter-departure time serves as an upper bound for the reordering ratio. The analytic model enables worst-case considerations more easily than with extensive simulations.

\section{Conclusion}

In this paper, we reviewed our queuing model for our reordering analysis presented in [7]. Applying the reordering queuing model, we stated that the reordering ratio of deterministic traffic with constant inter-arrival time is worse than any other traffic pattern in the same scenario. We proved the above statement using formal methods and showed illustrative results obtained from simulations.
In the simulation scenarios, we applied three different traffic characteristics (Poisson, Pareto and deterministic traffic) in three different network delay scenarios (geometric, linear and uniform distributed) to cover a broad spectrum of potential combinations of traffic and delay characteristics. The simulation results backup our findings and validated our proof.

The application of the model showing constant inter-arrival time enables reordering investigations, without performing exhaustive simulations, if the network delay distribution is known in advance (e.g. by previous simulations).

\section{ACKNOWLEDGMENT}

The work described in this paper was carried out with the support of the BONE-project ("Building the Future Optical Network in Europe"), a Network of Excellence by the European Commission through the 7th ICT-Framework Programme.

\section{REFERENCES}

[1] C. Qiao and M. Yoo, "Optical burst switching (OBS)—a new paradigm for an optical Internet," Journal of High Speed Networks, vol. 8, no. 1, pp. 69-84, Jan. 1999.

[2] A. Rahbar and O. Yang, "Contention avoidance and resolution schemes in bufferless all-optical packet-switched networks: a survey," Communications Surveys \& Tutorials, IEEE, vol. 10, no. 4, pp. 94-107, 2008.

[3] C. M. Gauger, M. Köhn, and J. Scharf, "Performance of contention resolution strategies in OBS network scenarios," in Proceedings of the 9 th Optoelectronics and Communications Conference/3rd International Conference on the Optical Internet (OECC/COIN), Yokohama/Japan, Jul. 2004.

[4] — - "Comparison of contention resolution strategies in OBS network scenarios," in Proceedings of the 6th International Conference on Transparent Optical Networks (ICTON), vol. 1, 2004, pp. 18-21.

[5] C. G. Argos, O. G. de Dios, and J. Aracil, "Adaptive Multi-Path Routing for OBS Networks," in Proceedings of the 9th International Conference on Transparent Optical Networks (ICTON), vol. 3, Jul. 2007, pp. 299302.

[6] L. Gharai, C. Perkins, and T. Lehman, "Packet reordering, high speed networks and transport protocol performance," Computer Communications and Networks, 2004. ICCCN 2004. Proceedings. 13th International Conference on, pp. 73-78, Oct. 2004.

[7] S. Gunreben, "An Optical Burst Reordering Model for a Time-based Burst Assembly Scheme," in Proceedings of the International Workshop on Optical Burst/Packet Switching 2008 (WOBS 2008), Sep. 2008.

[8] M. Allman, V. Paxson, and W. Stevens, "TCP Congestion Control," IETF, RFC 2581, Apr. 1999.

[9] X. Yu, J. Li, X. Cao, Y. Chen, and C. Qiao, "Traffic statistics and performance evaluation in optical burst switched networks," IEEE/OSA Journal of Lightwave Technology, vol. 22, no. 12, pp. 2722-2738, 2004. 
[10] S. Gowda, R. Shenai, K. Sivalingam, and H. Cankaya, "Performance evaluation of TCP over optical burst-switched (OBS) WDM networks," in Proceedings of the IEEE International Conference on Communications (ICC), vol. 2, 2003, pp. 1433-1437 vol.2.

[11] A. Detti and M. Listanti, "Impact of segments aggregation on TCP Reno flows in optical burst switching networks," in Proc. IEEE INFOCOM, 2002.

[12] F. Callegati, W. Cerroni, and C. Raffaelli, "Impact of Optical Packet Loss and Reordering on TCP Performance," in IEEE Global Telecommunications Conference (GLOBECOM '06), Nov. 2006, pp. 1-5.

[13] J. He and S. H. G. Chan, "TCP and UDP performance for Internet over optical packet-switched networks," Computer Networks, vol. 45, no. 4, pp. 505-521, Jul. 2004.

[14] J. P. Gelpke, M. Schlosser, E. Patzak, and H. Buchta, "Assessment of tcp performance in obs networks with load dependent contention," in Proceedings of the 9th International Conference on Transparent Optical Networks (ICTON), 2007.

[15] J. Perelló, S. Gunreben, and S. Spadaro, "A Quantitative Evaluation of Reordering in OBS Networks and its Impact on TCP Performance," in Proceedings of the IFIP Working Conference on Optical Network Design and Modelling (ONDM), Mar. 2008.

[16] M. Schlosser, E. Patzak, and P. Gelpke, "Impact of deflection routing on TCP performance in optical burst switching networks," in Proceedings of the 7th International Conference on Transparent Optical Networks (ICTON), vol. 1, Barcelona, Jun. 2005, pp. 220-223.

[17] N. Piratla, A. Jayasumana, and T. Banka, "On reorder density and its application to characterization of packet reordering," Local Computer Networks, 2005. 30th Anniversary. The IEEE Conference on, pp. 156165, 15-17 Nov. 2005.

[18] N. M. Piratla and A. P. Jayasumana, "Metrics for packet reordering-a comparative analysis," Int. J. Commun. Syst., vol. 21, no. 1, pp. 99-113, 2008.

[19] A. Morton, L. Ciavattone, G. Ramachandran, S. Shalunov, and J. Perser, "Packet Reordering Metrics," IETF, RFC 4737, Nov. 2006.

[20] S. Gunreben and G. Hu, "A Multi-layer Analysis on Reordering in Optical Burst Switched Networks," IEEE Communications Letters, vol. 11, no. 12, pp. 1013-1015, Dec. 2007.

[21] S. Gunreben, "Multi-layer Analysis to Quantify the Impact of Optical Burst Reordering on TCP Performance," in Proceedings of the 9th International Conference on Transparent Optical Networks (ICTON), 2007.

[22] W. Rudin, Real and complex analysis, 3rd ed. New York, NY, USA: McGraw-Hill, Inc., 1987.

[23] S. Bodamer, K. Dolzer, C. Gauger, M. Barisch, and M. Necker, IKR Simulation Library 2.6 User Guide, Institut für Kommunikationsnetze und Rechnersysteme, Universität Stuttgart, Jun. 2004. 